\title{
A Contrastive Study on Chinese Resultatives and Pseudo-resultatives
}

\author{
Xiaoxia Pan \\ College of Foreign Languages and Cultures \\ Sichuan University \\ Chengdu, China
}

\begin{abstract}
Among Chinese resultatives in existing researches, some resultatives do not express a resultative meaning. Based on the widely accepted definition of resultative in Chinese, this paper holds that some constructions, although in the same form as resultatives, are just pseudo-resultatives since there is no causality between the verb and the result. The contrastive study hereby reveals that resultatives and pseudo-resultatives in Chinese are different in semantics, analytic pragmatics, cognitive mechanism and motivation. Their complement adjectives also have different semantic properties.
\end{abstract}

Keywords-resultative; pseudo-resultative; causality; contrastive

\section{INTRODUCTION}

Resultatives in Chinese are special constructions, consisting of a verb and a complement which indicates a result. Unlike English resultatives, the two parts of Chinese resultatives are close in syntax, not allowing any syntactic elements between them. It is Lv (1980) who first named and defined resultatives or verb-resultative constructions in Chinese. Lv (1980: 11) defines Chinese resultatives as "the main verb plus an adjective or a verb that expresses the result". Later studies suggest that there are resultatives in a broad sense and resultatives in a narrow sense, but there is no clear and consistent definition for resultatives in a broad sense. According to Shi (2008: 5), "as far as resultatives in a broad sense are concerned, any adhesive verb-complement structure can be regarded as a resultative, even though some of the types do not represent the causative relationship between the action and the result". Although this view might solve some problems, this paper argues that there are considerable differences between causative resultatives and adhesive verb-complement constructions without causation, which need further study.

\section{RESUltatives AND PSEUDO-RESUltativeS}

Take a look at the following two example sentences:

(1) Guangdongren mai- gui- le hunan mi.

Cantonese buy-expensive- ASP Hunan rice

The Cantonese bought Hunan rice and as a result it became expensive.
(2) Zhongguo mai- gui- le eluosi shiyou.

China buy-expensive-ASP Russian oil

China bought Russian oil, which was too expensive.

In the two sentences, the syntactic structure of the construction "mai-gui (buy-expensive)" is both verb and adjective, but their propositional meanings are obviously different.

In (1) "mai-gui (buy-expensive)" expresses that the purchase of Hunan rice by the Cantonese has caused the price of Hunan rice to rise, while in (2) what it expresses is that the price of a certain quantity of oil that China buys from Russia is higher than the average oil price. These two categories can be tentatively distinguished as "mai-gui1" and "mai-gui2".

The question is whether the verb-complement construction can be treated as a resultative, or a verb-resultative construction (VRC). If "mai-gui" in either of the two sentences is a resultative, then why is there a semantic difference? Current research on Chinese resultatives does not thoroughly explain the semantic, pragmatic or cognitive mechanism of these constructions, which are different from resultatives. The differences between the two "mai-gui" still remain unexplained.

There is a consensus on the definition of resultatives in a narrow sense in the academic circle: a linguistic expression is a resultative if and only if its predicate expresses two events at the same time, that is, the action event and the result event; and the former causes the latter (Xiong, 2017; Cheng and Yang, 2016; Liu, 2014; Wang, 2011; Shi, 2008b; Wang and Wu, 2008).

According to this definition, "mai-guil" is a resultative, which consists of "buying rice" and "rice price rising" and the latter event is caused by the former. However, the "gui (expensive)" of "mai-gui2 (buy-expensive)" is not a result of "mai (buy)". That is to say, buying oil has not caused the oil price to rise.

Lu (1990, 2001) argues that this is a deviation from the expected result, which has become a view shared by some scholars (Song, 2004, 2006; Peng, 2006; Jiang, 2007; Shi, 2008a, 2013). However, this view will encounter a challenge when it is used to explain phenomena such as "mai-gui2 (buyexpensive)": In what sense is the causative relationship between the action event and the result event tenable? It is 
because this question is difficult to answer that such constructions can only be regarded as "special cases of resultative" (Shi, 2013, 2015) or "atypical resultatives" (Zhao, 2013). Therefore, this paper believes that this problem needs further study.

First of all, we need to explain why (1) and (2) are identical in form and syntax but different in meaning, and what are their respective cognitive mechanisms. Since this is a special case of resultative, we also need to explain what its semantic meaning and cognitive mechanism are. After all, "gui" in "mai-gui2" is also seen as a result. Therefore, the difference between the two results needs to be explained.

Secondly, "the expected result" is open to question, because "expected" does not exist semantically in the sentence. For example, in (1) it seems to imply the deviation of oil price from the buyer's expectation, but whether the buyer has an expectation cannot be determined semantically. However, buying oil and paying a higher price are a fact expressed semantically. In addition, in sentences such as the following:

(3) Zhe jian chenyi ni mai-gui- le.

This-CL shirt you buy-expensive-ASP

You have paid too much for this shirt.

"gui (expensive)" means not the deviation from the buyer's expectation, but the evaluation of the speaker.

Furthermore, by searching the Modern Chinese Corpus of Peking University and the Literature Corpus of Beijing Language and Culture University, 50 cases of "mai-gui" have been found, of which only 2 cases are "mai-gui1" and the rest are "mai-gui2". It can be seen that "mai-gui2" is an unmarked and typical usage in Chinese, which should be studied as an independent construction, rather than as a marginal and atypical resultative. As Shi (2015) points out: "strictly speaking, 'chi-zao (eat-early)' cannot be seen as a resultative, that is, it cannot be said that 'zao (early)' is caused by 'chi (eat)'. However, from the point of view of the integration mechanism and the coordination mode of argument structure, it can also be included into resultatives in a broad sense." This at least means that among the constructions regarded as resultatives there exist some which are identical in form yet different in meaning. This kind of constructions, which "cannot be regarded as resultatives" in a strict sense, are worthy of further investigation.

This paper refers to this kind of constructions, identical in form but different in meaning and function, as "pseudoresultative", and aims to conduct a contrastive study on Chinese resultatives and pseudo-resultatives from the perspectives of semantic nature, pragmatic inference, cognitive mechanism and motivation.

\section{SEMANTIC PROPERTIES OF ADJECTIVES IN RESUltATIVES AND PSEUDO-RESULTATIVES}

Just like resultatives, pseudo-resultatives are also verbcomplement structures. This paper argues that there are two indexes to judge whether a verb-complement construction is a resultative or a pseudo-resultative. Firstly, syntactically, the complement of a resultative can be a verb or an adjective while the complement of a pseudo-resultative is usually an adjective. What is more important is that their adjectives have different semantic properties and have different semantic relations with verbs. Adjectives that can be licensed into a resultative as a complement should have the following semantic characteristics: occurring after the verb, nonconstant and noninitial (Liu, 2014).

The "result" of a resultative is caused by the "verb". That is to say, the event expressed by the word "result" is caused by the event expressed by the predicate verb, which indicates that the character expressed by the complement adjective is a new character that the subject has experienced due to the action. In other words, what the complement adjective of the resultative expresses is the change of the attribute or state of the object involved in the action. The key point is that the attribute, state and its change expressed by the adjective can be logically determined and have a certain degree of objectivity.

For example:

(4) Ta ku-hong-le yanjing. she cry-red-ASP eyes

She cried and as a result her eyes became red.

(5) Linhui yi ku- shi- le shoupa.

Lin Hui already cry-wet-ASP handkerchief

Lin Hui had already cried the handkerchief wet.

The "hong (red)" in (4) indicates a change in the state of the eyes. Her eyes were not originally red, but later changed from not being red to being red because of crying. This is a semantically and logically determinable change of state. In addition, it is also an objectively ascertainable fact that the eyes are now either red or not. Similarly, "shi (wet)" in (5) is a determinable change of state: the handkerchief changes from being dry (not wet) to being wet because of the action event.

It is only when the word "result" expresses that the state it describes is caused by the action event and is logically determinable that it constitutes the result of the verb-resultative construction, which is subject to the logical stipulation of " $\mathrm{p} \mathrm{v}$ $\sim$ p".

The pseudo-resultative disobeys this stipulation. Compare the following two sentences:

(6) Tamen wa fangkongdong wa-kong-le shan. they dig bomb shelter dig-hollow-ASP mountain

They hollowed out the mountain by digging a bomb shelter.

(7) Tamen wa keng wa-qian-le

$$
\text { they dig pit dig-shallow-ASP }
$$

They dug a pit, yet the pit was too shallow.

In (6) the action of "digging" results in the "hollowness" of the mountain. Whether a mountain is "hollow" or not is a directly determinable empirical fact. A mountain is changed from "being not hollow" to "being hollow" because of "digging", which is semantically and logically determinable. 
But "qian (shallow)" in (7) is not the factual result caused by the action of "digging". First, the factual result of the action "digging" should be the fact that there was no pit and now there is one. A pit, of course, has the attribute of depth, but "qian (shallow)" does not express the attribute as a fact, but rather as an evaluative description of the attribute. An evaluative description of being deep or being shallow does not necessarily have an objective criterion and does not obey the logical stipulation of " $\mathrm{p} \mathrm{v} \sim \mathrm{p}$ ", yet it is relative to such factors as the speaker, the purpose or requirement of action, the environmental conditions, and so on.

A corresponding proof is that we can transform the sentence pattern of (7) with a certain modifier as follows:

(7a) Keng wa de tai qian le.

pit dig too shallow ASP

The pit was dug too shallow.

(7b) Keng wa de jiao qian.

pit dig relatively shallow

The pit was dug relatively shallow.

The same modification transformation fails to be applied to (6). Another convincing example is the following sentence:

(8) Zhe dao cai chao-xian-le

this-CL dish cook-salty-ASP

The dish was cooked and it was too salty.

The expectation of a chef is not to make the dish too salty. However, it is difficult to cater for all tastes. The dish cooked by a chef may be too salty for A, too light for $\mathrm{B}$, and just right for C, neither salty nor light. Obviously, "xian (salty)" is in essence a subjective evaluation of the dish by the speaker, not an objective attribute of the dish. It is impossible to obey the semantic logic of "salty v salty". If the complement in (7)'s verb-complement structure is also a kind of "result", then it is an evaluative result given by the individual speaker.

This phenomenon has also been noticed by other scholars. Wang (1996) calls this kind of complement "evaluation complement". Liao (2009) divides the resultative complements into status complements, evaluative complements and completion complements. However, this paper further argues that the verb-complement structure cannot be simply equated with the verb-resultative construction, because the complement of evaluation is not a result caused directly by the verb, and their relationship is not the verb-result relationship in the strict sense. The evaluative complement of a verb-complement structure usually expresses not the change of the object's attribute or state, nor the change of the attribute or state of the agent, but the evaluation made by the speaker. That is to say, the complement adjective of a pseudo-resultative expresses not a factual change of the attribute or state caused by the verb, but an evaluative description of the speaker.

This is consistent with Shi (2015): as for the category of "chao-xian (cook-salty)" and the category of "chi-zao (eat early)", their complements express a comment; the complement of the former category makes an indirect comment on the action through a comment on the object of the action, while the latter makes a direct comment on the action itself. But this paper argues that the complement not only gives an evaluative description of the action itself, but also presents a direct evaluative description of the object of the action.

\section{The Pragmatic InFERENCE Models OF THE VerB AND THE RESULT}

On further investigation, we find there are differences in pragmatic inferences of the verb and the result between resultatives and pseudo-resultatives. According to Brandom's "analytical pragmatics" theory, when people say a sentence, they carry out an inference behavior: they deduce the conclusion from the premise of other sentences, and continue to deduce the other conclusions from the premise of this conclusion (Brandom, 2008). Semantics lies in the structure of inference. In this way, different sentences have different meanings because of the different logical structures of inference.

Applying Brandom's pragmatic inference method to example sentences (1) and (2), we will get different models of discourse inference of "mai-gui (buy-expensive)":

(1') Guangdongren mai-gui-le hunan mi.

Cantonese buy-expensive-ASP Hunan rice

The Cantonese bought Hunan rice and as a result it

became expensive.

$\mathrm{xRy} \rightarrow \mathrm{Fy}$

(2') Zhongguo mai- gui- le eluosi shiyou.

China buy-expensive-ASP Russian oil

China bought Russian oil, which was too expensive.

$$
\mathrm{xRy} \wedge(\exists \mathrm{a}[\mathrm{a} \in \mathrm{y}] \wedge \mathrm{Fa})
$$

Obviously, (1') is a conditional expression whose logical relation of the verb and the result (VR) is causality: because $\mathrm{x}$ and $y$ have the relation $R$, so $y$ has the attribute of F; and (2') is essentially the conjunction of two propositional meanings. That is, the logical relation of its VR is: $\mathrm{x}$ has the relation $\mathrm{R}$ with $\mathrm{y}$; and there exists at least one "a", which belongs to y set, and "a" has the attribute F. When it comes to the example of this paper, we can do the following analysis.

First of all, in (1') the argument term y involved in "gui 1" is the universal term and the logical meaning is $\forall \mathrm{y}$; that is to say, "all Hunan rice" now has the attribute (F) of higher price than before. The premise of this assertion is that "Hunan rice was not expensive". At the same time, we can infer from (1) such an inference as: "now as long as everyone buys Hunan rice, they have to pay more money."

However, in (2') the argument term in "gui2" is a single term that refers to an element in a set; that is, only a single lot of Russian oil has been bought at a higher price. Not all Russian oil is expensive. The premise of (2') is obviously different from that of (1'). It is not that Russian oil was not expensive before and the price of Russian oil has risen because 
of the purchase by China. Instead, the premises can be that Russian oil sold to others is not expensive, or that Russian oil other than the one sold to China did not rise in price, and so on.

At the same time, taking (2') as a premise cannot reasonably deduce that "all countries must now pay more for Russian oil as long as they buy it". The logical inference from (2') should be that "China should shop around for oil next time", and so on. Thus, it can be seen that the semantic logical relationship between the verb "mai (buy)" and "gui (expensive)" in (1') and (2') requires different premises of inference, and the subsequent inferences are also different. According to analytical pragmatics, (1) and (2) are different in inference premise and subsequent inference. Although the two sentences have the same syntactic form, they are completely different in semantics, and this difference is pragmatically determined.

This paper argues that the logical distinction of VR semantic relations actually reinforces the previous discussion on the semantic attributes of adjectives. Let us look at two more examples:

(9) Ni mai-gui2- le, zhe jian zhi you 35 yuan.

you buy-expensive-ASP this-CL only have 35 yuan

You paid too much. This one was only worth 35 yuan.

(10) Fan zuo shao le.

rice cook little ASP

The rice cooked is too little.

In (9), "gui (expensive)" in "mai-gui2 (buy-expensive)" expresses that something purchased is expensive. It is an evaluation of the price of what is purchased, an evaluation presented by the speaker in contrast to the prices of other similar goods. In sentence (10), "zuo-shao (cook-little)" is an evaluation of the amount of rice cooked.

The semantic logic of the verb and the complement in either of the two sentences is not causality, so it does not express the meaning of resultative. That is to say, the price of something bought does not become higher because of the buying action, and the quantity of rice is not reduced because of the action of cooking. These two sentences are not a description of the change in the attribute of $y$, because different speakers are quite likely to give different, or even opposite, evaluations.

The premise of (9) can be that "both Sam and I bought one, yet we paid less money than you", or that "I found the same product in other stores was not as expensive as the one you bought", and so on. In (10) the premise of inference might be that "some people are not full", or that "those who come late have no rice to eat", and so on. There can be many subsequent inferences, which will not be stated in detail. But they do not involve the attributes of the object of the verb.

A more typical example of this is as follows:

(11) Wanfan chi-zao-le. dinner eat-early-ASP

\section{We had an early dinner.}

The object of the verb "chi (eat)", namely "dinner", has not undergone any change in its attributes, and the adjective "zao (early)" is not an attribute of dinner, nor is it a change in dinner's attributes. The premise of its inference should be a comparison with the regular dinner time. And its subsequent inference might be that you need an extra meal in the evening, or something. This is completely different from the semantic connotation of resultative, that is, "a change (R) of the attribute or state of a certain entity due to a certain action (V)".

There is causality between the verb event and the result event in a resultative, and causality has two characteristics, namely co-variation and temporality. Co-variation means that the result changes when the cause changes, and when the cause does not change the result does not change either. Temporality requires that the cause precedes the result. Therefore, the state expressed by the "result" of a resultative must appear after the "verb" (Xuan, 2017). The complement adjective of a pseudoresultative is an evaluative description of the action itself or the object involved in the action, so it can either appear after or with the "verb", but not necessarily co-variant. For example, in (11) "zao (early)" can be an evaluation of the action during and after a meal; in (7) "qian (shallow)" can be an evaluation of the pit after it has been dug or while it is being dug.

The above analysis from the perspective of analytical pragmatics makes it further clear that pseudo-resultatives have the same form as resultatives but are different from resultatives in semantics. Next, this paper will make a contrastive study on the cognitive mechanisms of resultative and pseudo-resultative, and then explore the generation motivations of pseudoresultatives.

\section{The Cognitive Mechanisms of Resultative AND PSEUDO-RESULTATIVE}

Modern cognitive linguistics regards language as a way of recognizing the world. Therefore, the generation mechanism of a language phenomenon should be investigated from the perspective of cognition. According to the aforementioned semantic analysis, the cognitive mechanism of pseudoresultative should be different from that of resultative.

The result of resultative refers semantically to the agent or the object of the action, and the subject of the result event is either a participant of the cause event or not. Accordingly, we can use two basic schemata to represent the semantic structures of resultative and other semantic structures can derive from the two basic schemata. Since there are so many categories of resultative and this paper is limited in length, we can't go into details here, just taking the following two as examples:

(12) Wo da- sui- le beizi.

I break-broken-ASP cup

I broke a cup into pieces.

(13) Wo ku-shi-le shoupa.

I cry-wet-ASP handkerchief

I cried and as a result the handkerchief became wet. 
Example (12) consists of two events, one is that "wo da beizi (I break a cup)" and the other is that "beizi sui le (the cup is broken)". The subject of the latter is also the patient of the former. It is obvious that there is causality between the two events. In (13) there also exist two events, that is, "wo ku (I cry)" and "shoupa shi le (the handkerchief becomes wet)". The subject of the latter event does not appear in the former, yet there is still semantic causality between them. The handkerchief becomes wet as a result of my crying. To be more specific, tears are a concomitant phenomenon of crying and it is tears that cause the handkerchief to become wet. However, a concomitant phenomenon can only be supervenient to an event and it cannot exist independently (Davidson, 2006: 105-121; $\mathrm{Xu}, 2015)$. In any case, the causative relationship between the action event and the result event is the cognitive schema of resultative.

Pseudo-resultative presents a different cognitive schema. The evaluation expressed by the complement is not made by the agent in the sentence, but by the speaker, who is outside the sentence itself. Therefore, the criterion held by the speaker becomes the criterion of the evaluation. We are to examine two pseudo-resultatives:

(14) Zhangsan wa keng wa-qian-le.

Zhangsan dig pit dig-shallow-ASP

Zhangsan dug a pit and the pit was too shallow.

(15) Lisi chi wanfan chi-zao-le.

\section{Lisi eat dinner eat-early-ASP}

Lisi had a dinner and it was too early for a dinner.

There is one important difference in the cognitive schema of pseudo-resultative: the evaluation given by the speaker is not at the same cognitive level as the event. Dimensions, such as "shen (deep), qian (shallow), zao (early) and wan (late)", do not belong to the cognition of the agent herself, but belong to the evaluation dimensions by the speaker who is not linguistically in the sentence. These dimensions are at a higher cognitive level than the factual level in which the agent is located. They constitute a possible three-dimensional cognitive schema which is not only related to the factual level, but also goes beyond the fact itself.

According to this schema, in (14) the patient "keng (pit)" of the action event "Zhangsan wa keng (Zhangsan dug a pit)" is what the complement is about. Whether something is deep or shallow, there is no maximum or minimum. They are openscale adjectives. The complement "qian (shallow)" of (14) is not an attribute or state of the agent of the action event, nor is it an objectively determinable attribute or state of the pit itself. It is actually the speaker's evaluation of the depth of the pit dug. In (15), the complement "zao (early)" describes neither the agent (Zhangsan) nor the patient (dinner) of the action event, but the action (eat). Likewise, "early" and "late" are open-scale adjectives and they are not an objectively determinable attribute or state of the action. The complement is an evaluation the speaker makes of the time at which the action occurs according to her chosen criterion. In the same way, this evaluation also comes from the speaker who is not the agent or patient at the linguistic and factual level and it is cognitively related. This connection is three-dimensional, because, after all, the two do not belong to the same cognitive level.

It is rather obvious that the cognitive schema of pseudoresultative is quite different from that of resultative. The cognitive schema of resultative presents a semantic structure at the same level, that is, what the complement expresses is at the same level with that of the action event. However, the cognitive schema of pseudo-resultative is kind of a threedimensional structure, two cognitive levels involved.

As a result, a key question must be answered: since the cognitive schemata of resultative and pseudo-resultative are quite different, then why can they take the same syntactic form? This is actually asking: what is the rationale behind the generation mechanism of pseudo-resultative?

At present, it is difficult to answer this question empirically and scientifically, so this paper intends to make the following speculative thinking. First, the proposition a sentence expresses can be distinguished in logical level. Inspired by Tarski (1999: 44-54), we follow his line of thought and can attribute the generation of pseudo-resultatives to the possibility that evaluative descriptions belong to a level of metacognition. That is, metacognition is not in the same level with real things and events, so it is not an objective grasp of the object itself and its attributes, states and so on. Since metacognition is a higherlevel evaluation of the perceived object, what it involves is not the object and the attribute, but the relationship between the event and the speaker, including her own expectation of the event, usually driven by intention. When the object of cognition is a concrete thing, then the object itself and its attribute, the change of attribute and so on are the contents of cognitive operation. Although metacognition is about a certain object, it does not regard the object itself as the cognitive object, but takes the fact, including the object, as the judging object and evaluates it.

The reason why pseudo-resultative is possible is that it is also "about" the object, but the speaker's cognitive structure is a three-dimensional "meta-"structure. Furthermore, the content of cognitive thinking can be different, but the structure is the same. An important point is that the syntactic features of thinking are the same, and the differences in levels are caused by the different natures of the objects in the thinking process (Liu, 2007). The difference of thinking level does not depend on syntax, but on the conceptual meaning of its processing.

From this point of view, both resultative and pseudoresultative are about specific objects, but the cognitive operation of resultative is about the object itself while the cognitive operation of the latter is about the relationship between the fact (including the object) and the speaker. Therefore, the predicate of a pseudo-resultative is not about what in fact the object (agent or patient) and its attributes are, but about how the speaker evaluates and judge the object and its attributes.

\section{CONCLUSION}

To sum up, this paper argues that a linguistic expression is a resultative if and only if the predicate of the expression expresses both the action event and the result event, and the 
two events have a causative relationship. A linguistic expression is a pseudo-resultative if and only if the predicate of the expression consists of an action event and an evaluative description of the action itself or the object involved in the action.

The causative relation between the verb and the result serves as the criterion for telling apart resultatives and pseudoresultatives. Because of this fundamental difference, between them there are a series of differences in semantics, semantic logic, cognitive motivation and analytic pragmatics.

\section{REFERENCES}

[1] R. Brandom, Between Saying and Doing: Towards an Analytic Pragmatism. New York: Oxford University Press, 2008.

[2] G. Cheng, D. Yang, "The word order of Mandarin resultative verb compounds and its related problems," Studies of the Chinese Language, vol. 5, pp. 526-540, 2016.

[3] D. Davidson, The Essential Davidson. New York: Oxford University Press, 2006

[4] H. Jiang, "An analysis of the multi-semantic-orientation in VCR complement," Journal of Anhui University, vol. 1, pp. 31-34, 2007.

[5] L. Levinson, "Arguments for pseudo-resultative predicates, "Natural Language \& Linguistic Theory, vol. 1, pp. 135-182, 2010.

[6] Y. Liao, "On the resultant complement used with resultant object in the verb-resultative construction," Journal of Henan Normal University (Philosophy \& Social Sciences), vol. 2, pp. 169-172, 2009.

[7] L. Liu, Raising Questions in and of Language. Chengdu: Sichuan University Press, 2007.

[8] Z. Liu, "The cognitive-semantic foundation for adjective to enter the verb-resultative construction," Chinese Language Learning, vol. 4, pp. 32-39, 2014.

[9] S. Lü, Eight Hundred Words in Modern Chinese (revised edition). Beijing: The Commercial Press, 1980/1999.

[10] J. Lu, "Semantic analysis on the verb-complement construction 'VAasp', “ Chinese Language Learning, vol. 1, pp. 1-6, 1990.

[11] J. Lu, "A further semantic analysis on the verb-complement construction 'VA-asp'," Chinese Language Learning, vol. 6, pp. 78-91, 2001.

[12] G. Peng, "The syntactic properties of the mandarin 'excessive resultative," Journal of Huazhong University of Science and Technology (Social Science Edition), vol. 4, pp. 47-51, 2006.

[13] C. Shi, "Multi-interface interactional mechanism of asymmetric distributions of verb-resultative constructions in construction groups," Chinese Teaching in The World, vol. 1, pp. 25-44, 2015.

[14] C. Shi, "More about the valence hierarchies of verb-resultative constructions and the issue of multivalence," Language Teaching and Research, vol. 5, pp. 65-74, 2013.

[15] C. Shi, Study on the Syntax and Semantics of Chinese Resultative Compounds. Beijing: Beijing Language University Press, 2008a.

[16] C. Shi, "The syntactic and semantic analysis of the VRC V-lei and its theoretic implication," Linguistic Sciences, vol. 3, pp. 242-258, $2008 \mathrm{~b}$.

[17] W. Song, "The distribution of verb-resultative constructions in several syntactic structures," Linguistic Researches, vol. 3, pp. 13-19, 2004.

[18] W. Song, "On verb-resultative constructions with a preposed object," Chinese Linguistics, vol. 4, pp. 57-66, 2006.

[19] A. Tarski, "The Semantic Conception of Truth and the Foundations of Semantics," in Modern Philosophy of Language, M. Baghramian, Ed. Washington D.C.: Counterpoint, 1999, pp. 44-54.

[20] H. Wang, "What is the semantics of verb-resultative construction," Chinese Language Learning, vol. 1, pp. 24-27, 1996.

[21] J. Wang, Y. Wu, "A review of researches on Chinese verb-resultative constructions in the perspective of generative grammar," Modern Foreign Languages, vol. 2, pp. 203-211, 2008.
[22] Y. Wang, "The progressive of RVCs in mandarin Chinese," Linguistic Sciences, vol. 1, pp. 70-82, 2011.

[23] X. Xiong, "A content-in-form-out analysis of the basic resultative construction," Modern Foreign Languages, vol. 2, pp. 147-156, 2017.

[24] S. Xu, "Metaphor from the perspective of mental-physical supervenience," Foreign Languages, vol. 4, pp. 2-11, 2015.

[25] Y. Xuan, "A typological analysis of the telic category of verbs," Chinese Teaching in The World, vol. 1, pp. 20-35, 2017.

[26] Q. Zhao, "The argument structure analysis of atypical Chinese resultative constructions," Chinese Language Learning, vol. 3, pp. 37-44, 2013. 